\title{
Evaluation of changes in myocardial perfusion and function on exercise in patients with coronary artery disease by gated MIBI scintigraphy
}

Philip Avery, Norah Hudson, Peter Hubner

\begin{abstract}
Objective-To investigate the ability of gated methoxy-isobutylisonitrile (MIBI) scintigraphy to measure changes in myocardial function as well as perfusion with exercise.

Setting-Regional cardiothoracic centre. Patients -43 presenting with chest pain, 28 with coronary artery disease on angiography, and 15 with normal coronary arteriograms.

Results-Gated perfusion images showed an improvement in detecting regions with stenosed arteries compared with non-gated images $(38 / 55 v 31 / 55, p \leqslant$ 0.01)). Functional analysis showed an increase in fractional shortening of $4 \cdot 11 \%$ in subjects with normal coronary arteries, whereas in those with coronary disease a fall of $0.57 \%$ was found $(p \leqslant 0.01)$. Both perfusion and function imaging showed an improved sensitivity compared with standard exercise testing $(p \leqslant 0.01)$. When both function and perfusion imaging were analysed all patients with coronary disease were detected. There was agreement in abnormal regions in 33/55 territories supplied by a stenosed artery. Combined perfusion and function detected 49155 (89\%) of abnormal regions, thus improving the overall sensitivity from $38 / 55(69 \%)$ by perfusion imaging alone ( $p \leqslant 0.01)$.

Conclusions-Gated methoxy-isobutylisonitrile scintigraphy can successfully evaluate perfusion and function on exercise, so improving the diagnostic usefulness of this agent.
\end{abstract}

(Br Heart f 1993;70:22-26)

Nuclear techniques have enabled the assessment of myocardial perfusion and regional and global cardiac function to determine the site, extent, and severity of coronary artery disease. Exercise myocardial perfusion imaging $^{1}$ and radionuclide ventriculography ${ }^{2}$ have provided useful information about the functional significance of coronary artery, stenoses. Some workers have added the results of these approaches to produce a highly sensitive method of detecting coronary artery disease. ${ }^{3-5}$ Moreover, assessment of ejection fraction with exercise has also been shown to be a most useful predictor of prognosis in coronary artery disease. ${ }^{67}$

Methoxy-isobutylisonitrile is a myocardial imaging agent with characteristics that have allowed evaluation of both perfusion and function. Both first pass ventriculography ${ }^{8}$ and gated imaging ${ }^{9}$ are reliable. We have evaluated the use of gated methoxy-isobutylisonitrile scintigraphy, and compared it with coronary angiography, to assess abnormalities in perfusion and regional wall motion with exercise for the detection and location of coronary artery disease.

\section{Patients and methods \\ PATIENTS}

Patients were recruited from referrals to the regional cardiothoracic centre. Entry criteria were chest pain with stable symptoms, New York Heart Association grades I and II, with no evidence of full thickness myocardial infarction on the electrocardiograph. Exclusions were severe symptoms, significant valvar or congenital heart disease and women of child bearing potential. All current medication was continued during investigations except for $\beta$ blockers that were discontinued for 48 hours before exercise testing.

We studied 43 patients (aged 31-75 (13 women and 30 men).

All patients gave written informed consent to the study and approval of the local ethics committee was obtained.

\section{CORONARY ANGIOGRAPHY}

Selective coronary angiography was performed by the Judkins technique with multiple views and was reported by two experienced observers. A significant stenosis was classified as $50 \%$ diameter loss or $70 \%$ cross sectional area loss in a major vessel supplying more than $20 \%$ of the myocardium.

These patients had radionuclide imaging 0 within one month of angiography. Subjects 0 were excluded if a significant cardiac event $\mathbb{D}$ had occurred-that is, myocardial infarction or unstable angina or if there had been a noticeable deterioration in their symptoms between angiography and radionuclide investigations.

EXERCISE PROTOCOL

Subjects exercised on a bicycle ergometer in the upright position. During exercise con- 윽 tinuous electrocardiographic monitoring was performed. A 12 lead electrocardiogram and blood pressure were recorded every three minutes during the test, at the end of the test, and every three minutes during recovery.

The test was ended by symptoms, $3 \mathrm{~mm}$ 


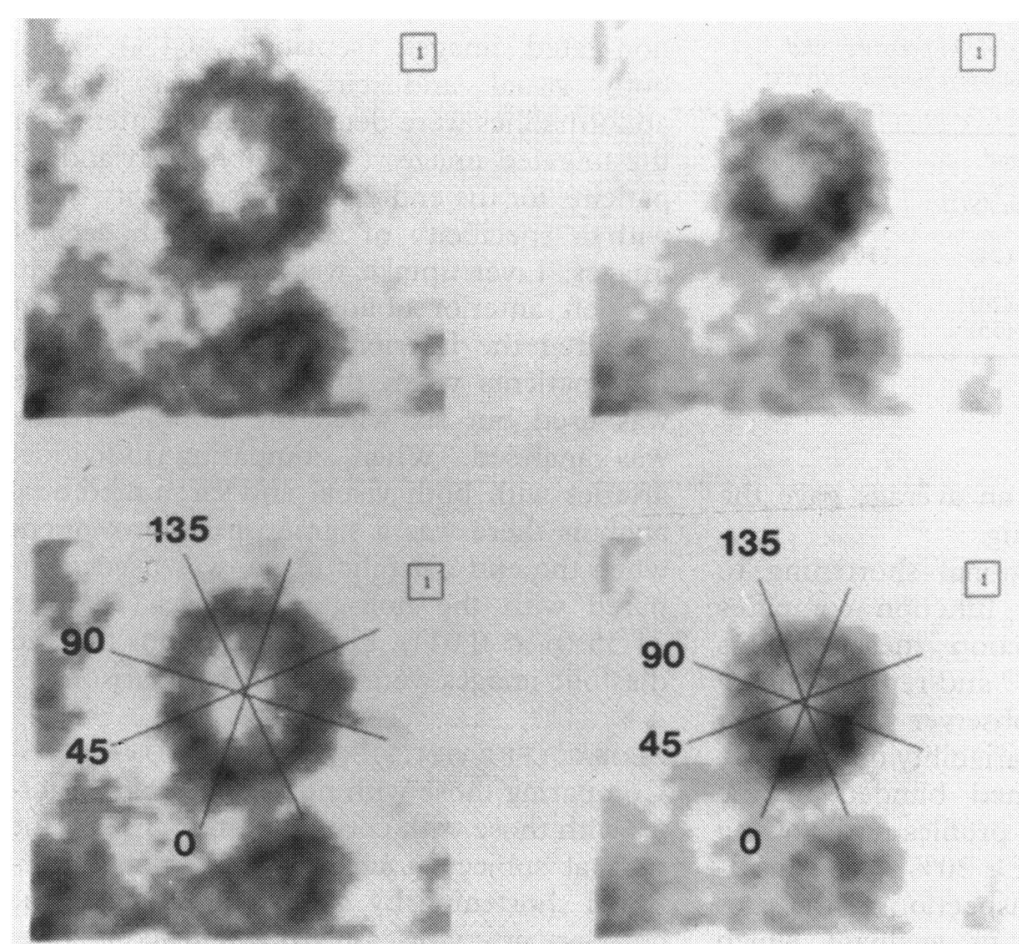

Figure 1 (Top) End diastolic and end systolic frames showing myocardial motion; (bottom) the same images with the four myocardial axes superimposed.
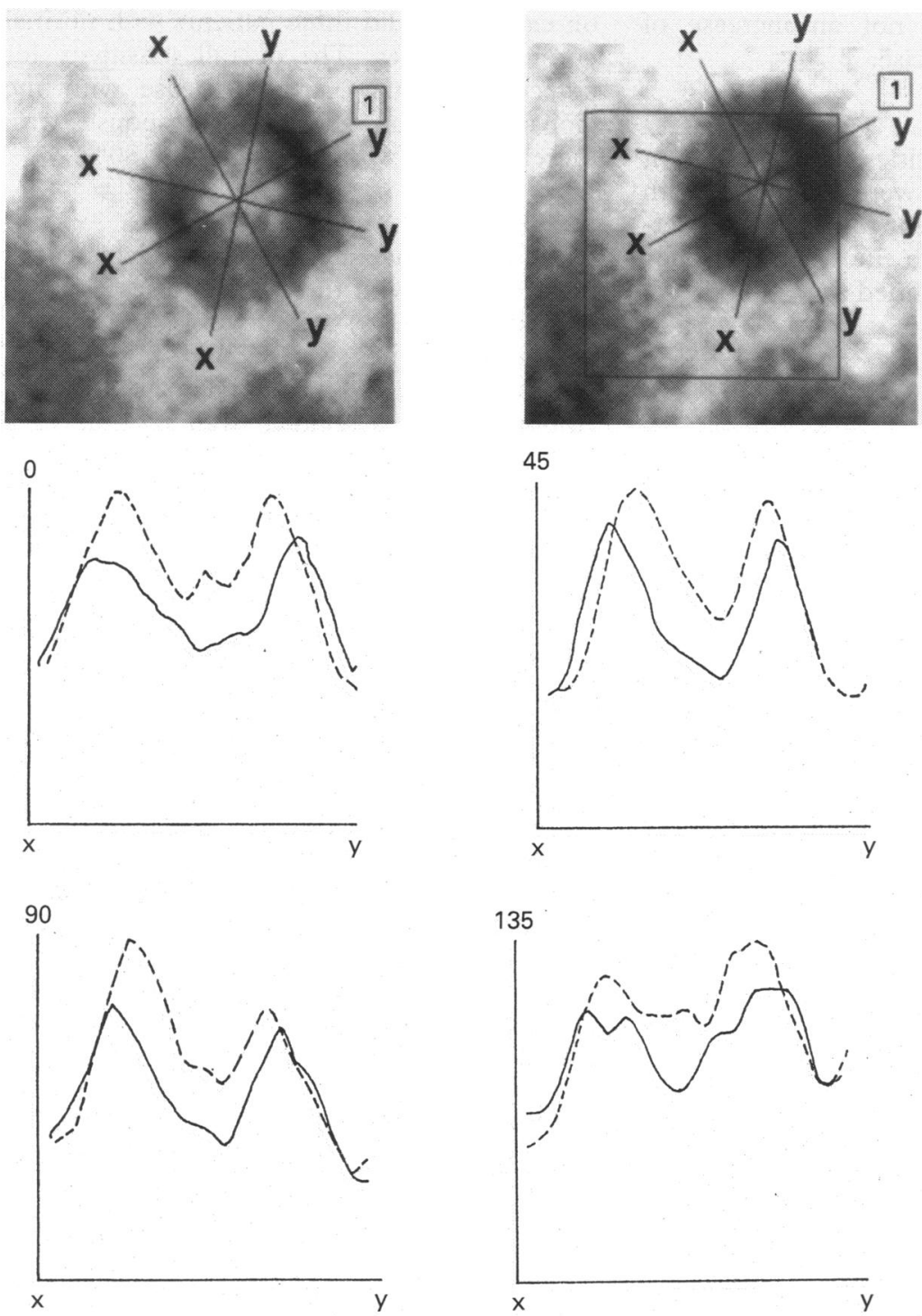

Figure 2 (Top) End diastolic and end systolic frames with the myocardial profile graphs generated by computer; (bottom) the solid line represents diastole and the dotted line systole. Contraction is seen as the peaks moving together. horizontal ST segment depression, progressive fall in systolic blood pressure of $>20$ $\mathrm{mm} \mathrm{Hg}$, ST segment elevation, or arrhythmia. A positive test was defined as $>1.5 \mathrm{~mm}$ horizontal or down sloping ST segment depression in three successive complexes. At peak exercise technetium-99m methoxyisobutylisonitrile $(500 \mathrm{MBq})$ were injected intravenously and images were acquired one hour later. Rest images were taken the next day with an injection of $1000 \mathrm{MBq}$ methoxyisobutylisonitrile. Images were again taken one hour later.

IMAGING PROTOCOL

Sixteen frame, gated images were taken on a single crystal mobile gamma camera with a parallel hole, low energy all purpose collimator. Data was stored on computer. Three standard projections were used left anterior oblique $40^{\circ}$, anterior, and left anterior oblique $70^{\circ}$. Non-gated images were made by summing all the 16 frames and an end diastolic image by summing frames 1 and 16 .

\section{ANALYSIS OF PERFUSION SCANS}

Summed 16 frame and end diastolic images were visually assessed blind by two observers. Defects scored according to site, severity (0, normal; 1 , mildly abnormal; 2 , considerably abnormal), and reversibility. These images were also analysed with circumferential analysis of $10^{\circ}$ segments to produce count graphs comparing exercise and rest. Quantitative analysis was defined as abnormal if there was $<70 \%$ uptake in any region, compared with $100 \%$ in the region of highest activity. Reversibility was defined as a relative increase of $20 \%$ from rest to exercise in any abnormal region.

\section{METHOXY-ISOBUTYLISONITRILE FUNCTION IMAGING}

After the rest perfusion scan the patient again sat upright on the bicycle ergometer with the camera angled in the left anterior oblique $40^{\circ}$ projection. A rest gated image was then obtained. The patient then exercised with the same protocol as before and gated images were taken during the last 2.5 minutes of each stage.

\section{ANALYSIS OF METHOXY-ISOBUTYLISONITRILE} FUNCTION SCANS

A computer program was devised to measure fractional shortening with count profile graphs in four axes at $0^{\circ}, 45^{\circ}, 90^{\circ}$, and $135^{\circ}$. The number of pixels between count peaks (pixel distance) at end diastole minus the pixel distance at end systole (ES) divided by the pixel distance at end diastole (ED) giving a figure for the fractional shortening (FS) along that particular axis.

$$
\text { FS }=\frac{\text { pixel distance ED }- \text { pixel distance ES }}{\text { pixel distance ED }}
$$

End diastolic and end systolic frames were selected and the profiles superimposed over them (fig 1). The computer then generated systolic and diastolic profiles along each axis (fig 2). The fractional shortening in each axis 
Comparison of heart rate, systolic blood pressure, and double product at peak exercise between normal subjects and those with coronary disease

\begin{tabular}{lll}
\hline & $\begin{array}{l}\text { Normal } \\
\text { subjects } \\
\text { Mean (SD) }\end{array}$ & $\begin{array}{l}\text { Patients with } \\
\text { coronary disease } \\
\text { Mean (SD) }\end{array}$ \\
\hline $\begin{array}{l}\text { Heart rate (beats/min) } \\
\begin{array}{l}\text { Systolic blood pressure } \\
\text { (mm Hg) }\end{array}\end{array}$ & $133(24)$ & $116(28)$ \\
\begin{tabular}{l} 
Double product \\
\hline
\end{tabular} & $238(20)$ & $166(19)$ \\
\hline
\end{tabular}

${ }^{\star} \mathrm{p}<0 \cdot 02$. Values are mean (SD).

was then displayed and an average gave the global fractional shortening.

This method of fractional shortening to evaluate left ventricular function compares well with ejection fraction measurements from blood pool studies, ${ }^{10}$ and reproducibility studies showed an interobserver variability of $<7 \%$ and intraobserver variability of $<5 \%$.

Analysis was performed blinded to the results of other tests and profiles not showing a fractional shortening of $>20 \%$ were considered abnormal. Visual inspection of the profiles made it possible to evaluate which myocardial regions of the profiles were responsible for the abnormal contraction. Exercise fractional shortening was defined as abnormal if there was not an increase of $>2.5 \%$ from rest to exercise.

\section{STATISTICAL ANALYSIS}

Sensitivities and specificities for the different methods of assessment were compared with McNemar's test. Different responses to exercise were compared with the Mann-Whitney test. Significance was defined as $\mathrm{p}<0.05$.

\section{Results}

\section{CORONARY ANGIOGRAPHY}

Twenty eight patients had appreciable coronary artery disease; eight had three vessel, 11 had two vessel, and nine had single vessel disease. Fifteen patients had normal coronary arteriograms.

Angiography detected 55 abnormal arteries; 20 significant left anterior descending artery, 14 circumflex artery, and 21 right coronary artery lesions.

\section{PERFUSION IMAGING}

Abnormalities were detected visually in the perfusion scan in 18 of 28 patients with coronary artery disease with both the gated and

Figure 3 Relative sensitivity, specificity, and predictive accuracy for coronary artery disease of electrocardiograph (ECG) stress testing, methoxyisobutylisonitrile perfusion imaging (PERF), methoxy-isobutylisonitrile regional wall motion evaluation ( $R W M)$, and combined methoxyisobutylisonitrile assessment.

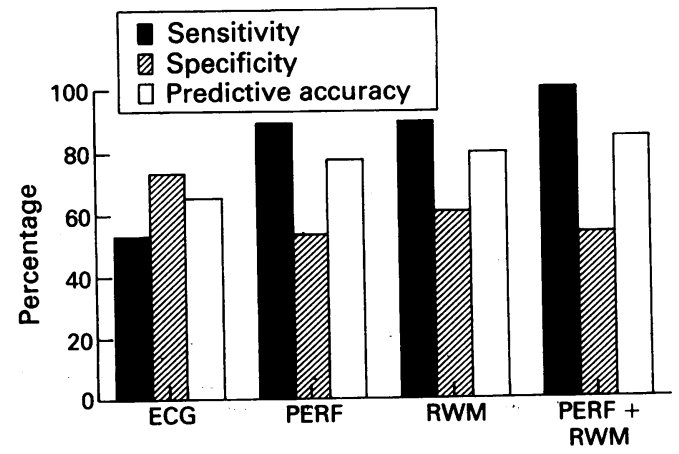

non-gated images (sensitivity 64\%). With both visual and circumferential analysis abnormalities were detected in 22 patients for the ungated images (sensitivity $78 \%$ ) and 25 patients for the end diastolic (sensitivity 89\%) with a specificity of $57 \%$ for both sets of images. Liver uptake was only a problem in the left anterior oblique projection when it obscured the inferior wall; this occurred in four patients when the end diastolic frame $c$. was used but six when the summed frame was analysed. When comparing individual arteries with both visual and circumferential analysis there was a significant improvement when the end diastolic image was used compared with the non-gated image (38/55 $v$ $31 / 55(p \leqslant 0.01)$ ). For these reasons the end diastolic images were used for comparison.

\section{GLOBAL LEFT VENTRICULAR FUNCTION}

Comparing those with normal coronary arteries with those with coronary artery disease the normal subjects increased their global fractional shortening by $4 \cdot 11 \%$ on exercise, the $N$ coronary artery disease group showed a fall in 은 fractional shortening of $0.57 \%(p \leqslant 0.01)$. Twenty patients with coronary disease, did $\sqsubset$ not show an increased fractional shortening on exercise as did three patients with normal $\vec{\theta}$ coronary arteries. The overall sensitivity for detecting coronary artery disease with the global fractional shortening response was, therefore, $71 \%$ with a specificity of $80 \%$.

REGIONAL WALL MOTION

Region fractional shortening was detected all $\bar{\partial}$ eight patients with three vessel disease, 10 of 3 11 patients (91\%) with two vessel disease, and abnormalities were found in seven of nine patients $(78 \%)$ with single vessel disease. Abnormalities in regional wall motion were also found in six of 15 patients (40\%) with normal coronary arteries by the fractional shortening method. The sensitivity of region- $\delta$ al fractional shortening, therefore, was $89 \%$ with a specificity of $60 \%$.

\section{COMPARISON OF NON-INVASIVE TESTS}

The maximum work load achieved, as $\tilde{\sigma}$ assessed by the double product of heart rate and systolic blood pressure, was significantly $N$ higher in the normal subjects than those with $\omega$ coronary disease (table). Sixteen of 28 穴 patients with coronary artery disease had a

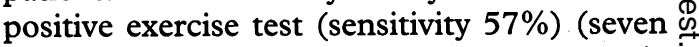
of eight patients with three vessel disease, six 0 of eleven patients with two vessel, and four of 0 nine patients with single vessel disease). This $\stackrel{\odot}{\overparen{D}}$ compares with methoxy-isobutylisonitrile per- $\stackrel{1}{2}$ fusion imaging with an overall sensitivity of $89 \%(25 / 28)$ and methoxy-isobutylisonitrile function imaging with a sensitivity of $89 \%$. 응 This showed a significant improvement in sensitivity with both perfusion and function imaging over electrocardiographic exercise testing $(p \leqslant 0.01)$.

The combination of myocardial perfusion and function imaging identified all the patients with coronary artery disease with a specificity of $53 \%$ (fig 3 ). 
Figure 4 Comparison of methoxy-isobutylisonitrile techniques in the 55 regions supplied by a stenosed coronary artery.

Abbreviations as for fig 3; + , positive; -, negative.

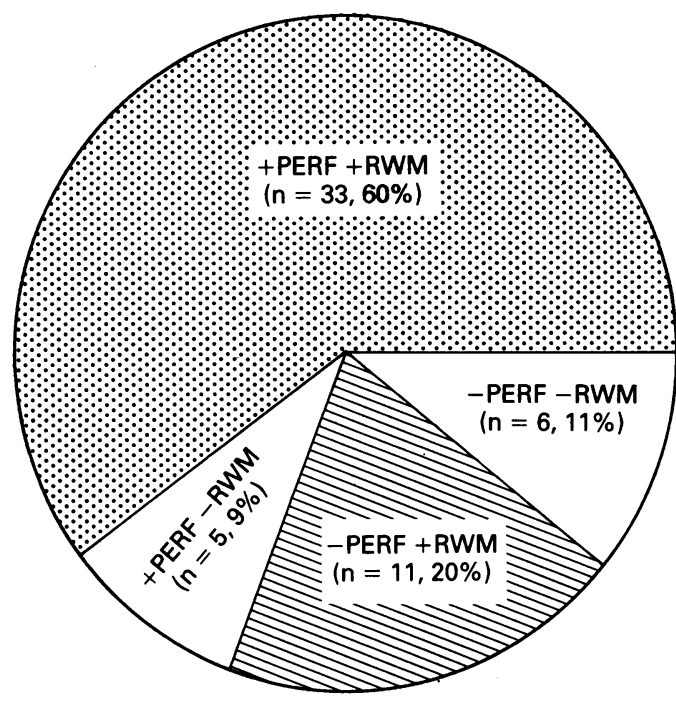

Forty four of the 55 regions supplied by the abnormal arteries were identified by analysis of new regional wall motion abnormalities on exercise and 38 of the 55 by abnormalities in exercise perfusion (NS). There was agreement in 33 regions having abnormalities in perfusion and function. The addition of regional wall motion abnormalities on exercise to perfusion defects found increased the abnormal regions detected by methoxy-isobutylisonitrile from 38 to 49 ( $\mathrm{p} \leqslant 0.01$, fig 4 ).

There were five regions with normal regional wall motion but perfusion defects on exercise; one in the left anterior descending region, one in the circumflex, and three in the right coronary artery. Eleven regions had normal perfusion but exercise induced wall motion abnormalities; four in the left anterior descending region, five in the circumflex, and two in the right coronary artery.

\section{Discussion}

Experimental studies have shown a correlation between coronary artery stenosis and myocardial contraction with dysfunction occurring before electrocardiographic abnormalities. ${ }^{11}$ Bodenheimer et al showed a close correlation between severity of coronary stenosis, decreased myocardial perfusion, and contraction in humans. ${ }^{12}$ Glyceryl trinitrate contrast ventriculography showed reversible wall motion abnormalities in most patients with exercise induced perfusion defects. A similar correlation has been found between exercise induced abnormalities of regional wall motion and perfusion defects. Johnstone et al found regional wall motion abnormalities in 21 of 24 patients with a defect identified by thallium imaging on exercise, ${ }^{5}$ and similar results have been found by other workers. ${ }^{413}$

Improved sensitivity for detecting coronary artery disease has been shown by abnormalities of exercise function compared with perfusion imaging. ${ }^{45}$ Single photon emission computerised tomography perfusion imaging, however, has been suggested to be the most sensitive method of detecting coronary artery disease in patients with an equivocal electrocardiographic stress test, ${ }^{14}$ but this is not available in all centres.

The combination of perfusion and function has been shown to improve sensitivity over planar perfusion imaging alone. Caldwell et al showed $100 \%$ sensitivity with a $56 \%$ specificity ${ }^{4}$ and Bodenheimer and coworkers $96 \%$ sensitivity and $58 \%$ specificity with both perfusion and function imaging. ${ }^{3}$ The low specificity in these studies contrasts with work by Jengo et al who, despite showing a high sensitivity (98\%) also showed a high degree of specificity $(94 \%)^{13}$ and Johnstone et al with a sensitivity of $92 \%$ and specificity of $100 \% .5$ These differences may represent a contrast in patient selection between normal controls and a control group of patients with chest pain and normal coronary arteries and to different interpretation of abnormalities of regional wall motion.

Because of a low photon energy, gated thallium scintigraphy would not allow adequate count statistics for quantitative analysis. The introduction of technetium ligands has. This study of combined methoxyisobutylisonitrile perfusion and function imaging has shown a correlation between regional wall motion abnormalities found by regional fractional shortening measurements and perfusion defects induced by exercise similar to previous studies. Of the 38 regions with perfusion abnormalities 33 regions had abnormalities of regional wall motion.

Fractional shortening responses indicated a greater number of abnormal regions than perfusion imaging. Forty four of $\mathbf{5 5}$ regions supplied by an artery with a significant stenosis had regional wall motion abnormalities and 38 of 55 had exercise induced perfusion defects but this did not reach significance. The improvement in detection of coronary artery disease with wall motion analysis was in the territories of the left anterior descending and left circumflex arteries. The improvement in detection of a significant circumflex lesion by regional dysfunction has been noted before $^{15}$ and combined imaging may be a particularly useful assessment of this region. There was also an improved sensitivity (89\%) in detecting coronary artery disease, with both perfusion and function imaging compared with standard exercise testing, only 16 of 28 patients showed a positive electrocardiogram during stress (sensitivity 59\%).

The overall detection rate for patients with coronary artery disease was $100 \%$. This was associated with a low specificity $53 \%$. These results are similar to some of the other series of combined perfusion and function imaging already discussed. The sensitivity of the perfusion imaging compares well with other studies of planar imaging, ${ }^{16}$ and whereas a low specificity was also found it must be noted that four subjects in the control group of normal or trivial coronary artery disease on angiography had abnormalities in all of their non-invasive tests and may represent syndrome X. ${ }^{17}$ The specificities rise significantly 
to $73 \%$ if the results of these subjects were excluded from specificity calculations.

Bodenheimer et al also showed an improvement in detection of single vessel disease from $11 \%$ with the electrocardiogram alone to $44 \%$ with thallium imaging and $56 \%$ with regional wall motion abnormalities. ${ }^{3}$ We have shown a good detection rate for single vessel disease by evaluating regional wall motions by gated scintigraphy with an abnormality in all but one patient, with all patients having single vessel disease detected by combined imaging. This may be important for the assessment of patients undergoing coronary angioplasty as many have single vessel disease.

Despite this low specificity combined perfusion and function imaging could be performed as a screening test before coronary angiography in patients with chest pain. In this study poptrlation, $53 \%$ of the patients with normal coronary arteries-that is, $19 \%$ of the whole patient group-need not have been investigated with coronary angiography on the grounds of normal combined imaging.

Not only does the addition of myocardial function imaging improve the detection rate and accuracy of methoxy-isobutylisonitrile imaging but it may be important for estimation of prognosis in coronary artery disease and may indicate jeopardised myocardium.

Previous studies have shown a correlation between exercise function and subsequent cardiac events in coronary artery disease even in those without previous infarction, ${ }^{67}$ with the functional information from radionuclide angiography providing information that supplements coronary angiography..$^{18}$ As well as an increased number of cardiac events, survival has been shown to be poorer with poor left ventricular function with exercise, ${ }^{19}$ but patients with a greater number of exercise induced left ventricular dysfunctions also have a greater benefit from bypass surgery. ${ }^{20}$ Gated methoxy-isobutylisonitrile scintigraphy, by evaluating function and perfusion may, therefore, be useful in the assessment of prognosis and decision making before surgery as well as in the diagnosis.

Massie et al used combined perfusion and function imaging to demonstrate regions with asynergy at rest and exercise induced perfusion defects that, after revascularisation, regained normal contraction. ${ }^{21}$ Methoxyisobutylisonitrile would, therefore, be an ideal agent to assess jeopardised myocardium as perfusion and function could be evaluated simultaneously. Studies are underway with gated imaging to evaluate the changes in perfusion and function after revascularisation.

A limitation of the technique of measuring myocardial function is that contraction cannot be evaluated if there is no uptake in a particular region, as with a previous full thickness myocardial infarction, although other regions can be assessed with the other profile axes.

In conclusion, gated methoxy-isobutylisonitrile scintigraphy provides a sensitive detector of coronary artery disease that may also provide prognostic information in these patients and allow evaluation of jeopardised myocardium and adequacy of revascularisation without the need for coronary angiography in some patients.

1 Strauss HW, Harrison K, Langan K, Lebowitz E, Pitt B. Thallium-201 for myocardial imaging. Circulation 1975;51:641-5.

2 Borer JS, Bacharach SL, Green MV, Kent KM, Epstein $\mathrm{SE}$, Johnston GS. Real-time radionuclide cineangiography in the noninvasive evaluation of global and regional left ventricular function at rest and during exercise in patients with coronary-artery disease. $N$ Engl $f \mathrm{Med}$ 1977;296:839-44.

3 Bodenheimer MM, Banka VS, Fooshee CM, Helfant RH. Comparative sensitivity of the exercise electrocardiogram, thallium imaging and stress radionuclide angiography to detect the presence and severity of coronary graphy to detect the presence and severity

4 Caldwell JH, Hamilton GW, Sorensen SG, Ritchie JL Williams DL, Kennedy JW. The detection of coronary artery disease with radionuclide techniques: a comparison of rest-exercise thallium imaging and ejection fracion response. Circulation 1980;61:610-9.

Johnstone DE, Sands MJ, Berger HJ, et al. Comparison of exercise radionuclide angiocardiography and thallium201 myocardial perfusion imaging in coronary artery disease. Am $\mathcal{F}$ Cardiol 1980;45:1113-9.

6 Pryor DB, Harrell FE, Lee KL, et al. Prognostic indicators from radionuclide angiography in medically treated patients with coronary artery disease. Am f Cardiol 1984;53:18-22.

7 Iskandrian AS, Hakki AH, Goel IP, Mundth ED, KaneMarsch SA, Schenk CL. The use of rest and exercise radionuclide ventriculography in risk stratification in patients with suspected coronary artery disease. $\mathrm{Am}$ Hean $₹$ 1985;110:864-72.

8 Basi G, Sciagrar, Bull U, et al. Assessment of left ventricular function with first-pass radionuclide angiography using technetium- $99 \mathrm{~m}$ hexakis-2-methoxyisobutylisonitrile: a European multicentre study. Eur $\mathcal{F}$ Nucl Med 1991;18:178-83.

9 Najm YC, Timmis A, Maisey MN, et al. The evaluation of ventricular function using gated myocardial imaging with technetium-99m MIBI. Eur Heart ff 1989;10: 142-8.

10 Avery PG, Williams H, Early MY, Hudson NM, Hubner PJB. Gated ${ }^{99 m}$ Tc-MIBI myocardial function imaging. Nucl Med Commun 1991;12:677-85.

11 Battler A Froelicher VF, Gallagher KP, Kemper WS Ross J. Dissociation between regional myocardial dysfunction and ECG changes during ischaemia in the conscious dog. Circulation 1980;62:735-44.

12 Bodenheimer MM, Banka VS, Fooshee CM, Hermann GA, Helfant RH. Relationship between myocardial perfusion and the presence, severity, and reversibility of asynergy in patients with coronary artery disease. Circulation 1978;58:789-95.

13 Jengo JA, Freeman R, Brizendine M, Mena I. Detection of coronary artery disease: comparison of exercise stress radionuclide angiography and thallium stress perfusion imaging. Am $\mathcal{F}$ Cardiol 1980;45:535-41.

14 Candell-Riera J, Castell-Conesa J, Ortega-Alcalde D, Domenech-Torne FM, Soler-Soler J. Diagnostic accuracy of radionuclide techniques in patients with equivocal electrocardiographic exercise testing. Eur Heart f 1990;11:980-9.

15 Dilsizen V, Perrone-Filardi P, Cannon RO, Freedman NM, Bacharach SL, Bonow RO. Comparison of exercise radionuclide angiography with thallium SPECT imaging for detection of significant narrowing of the left circumflex coronary artery. Am f Cardiol 1991;68: 320-8.

16 Maisey $\mathrm{MN}$, Lowry A, Bischof-Delaloye A, et al European multi-centre comparison of thallium-201 and technetium-99m methoxyisobutylisonitrile in ischaemic heart disease. Eur $\mathcal{F}$ Nucl Med 1990;16:869-72.

17 Cannon RO, Bonow RO, Bacharach SL, et al. Left ventricular dysfunction in patients with angina pectoris, tricular epicardial coronary arteries and abnorma vasodilator reserve. Circulation 1985;71:218-26.

18 Lee KL, Pryor DB, Pieper KS, et al. Prognostic value of radionuclide angiography in medically treated patients with coronary artery disease: a comparison with clinical with coronary artery disease: a comparison with clinical and cathet $1705-17$.

19 Bonow RO, Green MV, Bacharach SL. Radionuclide angiography during exercise in patients with coronary artery disease: diagnostic, prognostic and therapeutic implications. Int $\mathcal{F}$ Cardiol 1984;5;:229-33.

20 Jones RH, Floyd RD, Austin EH, Sabiston DC. The role of radionuclide angiography in the preoperative prediction of pain relief and prolonged survival following coronary artery by-pass grafting. Ann Surg 1983;197: 743-53.

21 Massie BM, Botvinick EH, Brundage BH, Greenberg B, Shames D, Gelberg H. Relationship of regional myocardial perfusion and segmental wall motion: a physiological basis for understanding the presence and reversibility of asynergy. Circulation 1978;58:1154-63.

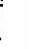

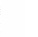

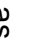

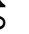

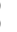

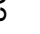

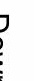
(D)

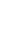
. .

\section{.} .

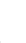

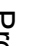

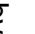

